\title{
Hyperbolicity of the 3+1 System of Einstein Equations
}

\author{
Yvonne Choquet-Bruhat ${ }^{1}$ and Tommaso Ruggeri ${ }^{2}$ \\ 1 Département de Mécanique, Université Paris VI, Paris, France \\ 2 Istituto di Matematica Applicata, Università di Bologna, Bologna, Italy
}

\begin{abstract}
By a suitable choice of the lapse, which in a natural way is connected to the space metric, we obtain a hyperbolic system from the $3+1$ system of Einstein equations with zero shift; this is accomplished by combining the evolution equations with the constraints.
\end{abstract}

\section{Introduction}

The success of the temporal gauge in Yang Mills theory for proving existence theorems (cf. $[17,18,7]$ ) has led us to look for an analogous gauge in general relativity. It is well known that neither normal gaussian coordinates, nor other arbitrary choices of lapse and shift lead to a hyperbolic evolution system for the conjugate unknowns $g_{i j}$ and $P^{i j}$. However this system is very useful in numerical computations of space time models [16] as well as in certain quantization procedures $([2,1])$.

We show here that if we call "temporal gauge" a choice of time lines orthogonal to the space sections (choice always possible in a globally hyperbolic manifold) the conjugate unknowns satisfy a hyperbolic evolution system if we choose the lapse appropriately. The local existence and global uniqueness theorems ${ }^{1}$, under their most refined form (cf. $[6,4]$ ) can then be deduced with the help of the usual machinery constraints - Bianchi identities. The explicit geometric expression of the evolution system gives a better light to study global problems.

Let us remark, for numerical relativists, that the choice of lapse we propose with zero shift, has the merit to make stable the solution of the Cauchy problem, a property which could be useful in their constructions of dynamical models.

\section{The 3+1 Equations}

We recall the Einstein equations in the $3+1$ form (see $[15,8,1]$ ), or the review article [9]). Let $S \times \mathbb{R}$ denote the space-time manifold; we choose the time-lines

1 A different approach which also does not use harmonic coordinates is due to DeTurk (preprint) that introduces an assigned 2-tensor 
orthogonal to the space sections (zero shift ${ }^{2}$ ). Then the space-time metric is given by

$$
{ }^{(4)} g_{\alpha \beta} d x^{\alpha} d x^{\beta}=-\alpha^{2}\left(d x^{0}\right)^{2}+g_{i j} d x^{i} d x^{j} \text {, }
$$

the "conjugate momentum" of $g_{i j}$ is the contravariant 2-tensor,

$$
\mathbf{P}=\mathbf{K}-\mathbf{g} \operatorname{tr} \mathbf{K}\left\langle>P^{i j}=K^{i j}-g^{i j} K_{r}^{r},\right.
$$

where $\mathbf{K}$ denotes the second fundamental form of the submanifold $t=$ const.

Putting $\dot{f}=\partial f / \partial x^{0}$, we have

$$
\dot{g}^{i j}=\alpha\left(2 P^{i j}-g^{i j} P\right),
$$

where $P=P_{k}^{k}$.

The Einstein equations in vacuum are

$$
\Sigma^{\alpha \beta} \equiv{ }^{(4)} R^{\alpha \beta}-\frac{1}{2}^{(4)} g^{\alpha \beta(4)} R=0 .
$$

The evolution part is given by $(i=1,2,3)$ :

$$
\Sigma^{i j} \equiv-\frac{1}{\alpha}\left(\dot{P}^{i j}+D^{i} D^{j} \alpha-g^{i j} \Delta \alpha\right)+L^{i j}+S^{i j},
$$

where

$$
\begin{gathered}
L^{i j} \equiv 2 P^{i k} P_{k}^{j}-\frac{3}{2} P P^{i j}-\frac{1}{2} g^{i j} P^{h k} P_{h k}+\frac{1}{4} g^{i j} P^{2}, \\
S^{i j} \equiv R^{i j}-\frac{1}{2} g^{i j} R .
\end{gathered}
$$

Here $R^{i j}$ and $R$ are the Ricci curvature and the scalar curvature of the space metric $g_{i j} ; D$ denotes the covariant derivative with respect to the metric $g_{i j}$ and $\Delta=g^{i j} D_{i} D_{j}$ is the usual Laplacian.

The constraint part, which does not contain second derivatives with respect to the time variable, is

$$
\begin{gathered}
\Sigma^{i 0} \equiv \frac{1}{\alpha} D_{j} P^{i j} \\
\Sigma^{00} \equiv \frac{1}{2 \alpha^{2}}\left(R-P^{i j} P_{i j}+\frac{1}{2} P^{2}\right) .
\end{gathered}
$$

\section{The Hyperbolic System}

Lemma. The following identity holds

$$
\dot{S}^{i j}+\alpha^{2}\left(D^{i} \Sigma^{j 0}+D^{j} \Sigma^{i 0}-g^{i j} D_{k} \Sigma^{k 0}\right) \equiv \alpha \Delta P^{i j}+\frac{1}{2}\left(D^{i} D^{j}-g^{i j} \Delta\right)(\alpha P)+H^{i j},
$$

where $H^{i j}$ is a given function of $g^{h k}, \alpha$, of their derivatives of order at most 2, of $P^{h k}$ and of its derivative of order at most 1 . Furthermore $H^{i j}$ is analytic if $g=\operatorname{det}\left(g_{i j}\right) \neq 0$.

2 Analogous results can be obtained with a nonzero given shift. For an intrinsic formulation of the evolution problem with an arbitrary shift, see the papers by G. Caricato (e.g. [3]), that used the technique of "projection" due to C. Cattaneo [4] 
Proof. A straightforward calculation shows that the principal terms - of order two in $P^{h k}$ - of the $x^{0}$ derivative of $S^{i j} \equiv R^{i j}-\frac{1}{2} g^{i j}$ are given by:

$$
\dot{S}^{i j} \simeq \alpha\left(\Delta P^{i j}-D^{i} D_{k} P^{j k}-D^{j} D_{k} P^{i k}+g^{i j} D_{h} D_{k} P^{h k}+\frac{1}{2}\left(D^{i} D^{j} P-g^{i j} \Delta P\right)\right) .
$$

Then by exploiting the identity (7) we obtain (9).

Theorem. The equations

$$
\dot{\Sigma}^{i j}+\alpha^{2}\left(D^{i} \Sigma^{j 0}+D^{j} \sum^{i 0}-g^{i j} D_{h} \sum^{h 0}\right)=0,
$$

with the relation (3) between $P^{i j}$ and $g^{i j}$, form a strictly hyperbolic system in the unknowns $P^{i j}$, and $g^{i j}$, if the lapse $\alpha$ is chosen such that it satisfies the equation

$$
\partial\left(\alpha^{-1} g^{1 / 2}\right) / \partial x^{0}=0 .
$$

The "dependence domain" of the solutions is determined by the isotropic cone of the space metric (1).

Proof. From (4) we get

$$
\dot{\Sigma}^{i j} \equiv-\frac{1}{\alpha} \ddot{P}^{i j}+\dot{S}^{i j}+\left(g^{i j} \Delta-D^{i} D^{j}\right)\left(\alpha^{-1} \dot{\alpha}\right)+M^{i j},
$$

where $M^{i j}$ depends only on $g^{h k}, \alpha$ and their derivatives up to the second order, $P^{h k}$ and their derivatives of order 1.

Taking into account (9) we get

$$
\begin{aligned}
& \dot{\Sigma}^{i j}+\alpha^{2}\left(D^{i} \Sigma^{j 0}+D^{j} \Sigma^{i 0}-g^{i j} D_{h} \Sigma^{h 0}\right) \\
& \equiv-\alpha \square P^{i j}+\left(g^{i j} \Delta-D^{i} D^{j}\right)\left(\frac{\dot{\alpha}}{\alpha}-\frac{\alpha}{2} P\right)+M^{i j}+H^{i j}
\end{aligned}
$$

where $\square$ is the hyperbolic operator:

$$
\square=\frac{1}{\alpha^{2}} \frac{\partial^{2}}{\partial x^{02}}-\Delta
$$

We note that if $\alpha$ is such that

$$
2 \alpha^{-2} \dot{\alpha}=P,
$$

then the principal terms in (13) reduce to $-\alpha \square P^{i j}$.

As $P=\alpha^{-1} g^{-1} \dot{g}$, Eq. (14) can be written

$$
g^{-1} \dot{g}=2 \alpha^{-1} \dot{\alpha},
$$

which, because of the condition $\alpha>0$, has the solution

$$
\alpha=e^{-1 / 2} g^{1 / 2},
$$

where $e=\operatorname{det}\left(e_{i j}\right), e_{i j}$ arbitrarily given Riemann metric on $S$. system

With this choice the system (3), (11) becomes a quasi-diagonal hyperbolic

$$
\begin{gathered}
\frac{\partial g^{i j}}{\partial x^{0}}=\left(e^{-1} g\right)^{1 / 2}\left(2 P^{i j}-g^{i j} P\right) \\
\square P^{i j}-\left(e g^{-1}\right)^{1 / 2}\left(M^{i j}+H^{i j}\right)=0,
\end{gathered}
$$


whose characteristics at a point are the isotropic cone and the time axis. This axis being interior to the cone, the cone determines the propagation.

Remark 1. The system can be written as a third order system in the unknowns $g g^{i j}$, with principal part $\square\left\{\frac{\partial}{\partial x^{0}}\left(g g^{i j}\right)\right\}$, because

$$
P^{i j}=\frac{1}{2 \alpha} \frac{\partial}{\partial x^{0}}\left(g g^{i j}\right)
$$

Remark 2. Condition (16) coincides, for the time-lines orthogonal to the space sections, with the harmonicity condition of time-like index:

$$
g^{\alpha \beta} \Gamma_{\alpha \beta}^{0}=0 .
$$

Remark 3. The hamiltonian constraint (8) cannot be used as the other constraint to obtain hyperbolicity: the principal part of $\dot{R}$ is a linear differential operator acting on the principal terms of the other constraints.

We need to verify that every solution of the system (17)-(18) is a solution of the Einstein system. This will be accomplished by use of the following:

Theorem 2. Let $g^{i j}$ and $P^{i j}$ verify the hyperbolic system (17), (18), then the Einstein tensor $\Sigma^{\alpha \beta}$, corresponding to the metric

$$
-\alpha^{2}\left(d x^{0}\right)^{2}+g_{i j} d x^{i} d x^{j}, \quad \alpha=\sqrt{g / e},
$$

verifies a linear hyperbolic homogeneous system.

Proof. By Bianchi identities ( $\nabla$ denotes covariant derivation with respect to the metric $g_{\alpha \beta}$ ) we have

$$
\nabla_{\alpha} \Sigma^{\alpha \beta} \equiv 0 .
$$

Equation (18) says that the metric $-\alpha^{2}\left(d x^{0}\right)^{2}+g_{i j} d x^{i} d x^{j}$ with $\alpha^{2}=e^{-1} g$, verifies the equations:

$$
\dot{\Sigma}^{i j}+\alpha^{2}\left(D^{i} \Sigma^{j 0}+D^{j} \Sigma^{i 0}-g^{i j} D_{h} \Sigma^{h 0}\right)=0 .
$$

Hence also

$$
D_{j}\left\{\dot{\Sigma}^{i j}+\alpha^{2}\left(D^{i} \Sigma^{j 0}+D^{j} \Sigma^{i 0}-g^{i j} D_{h} \Sigma^{h 0}\right)\right\}=0 .
$$

So using (19) we obtain:

$$
\square \Sigma^{i 0}+f^{i}=0,
$$

where $f^{i}$ is a linear form in $\Sigma^{\alpha \beta}$ and $D^{k} \Sigma^{h 0}$. The system formed by (20), (21) and the identity:

$$
\nabla_{0} \Sigma^{00}+\nabla_{i} \Sigma^{i 0} \equiv 0,
$$

is a strictly hyperbolic system in the unknowns $\Sigma^{\alpha \beta}$, analogous to the one of the preceding section, which moreover is linear and homogeneous. 


\section{Existence for Einstein Equations}

Let $S$ be a $C^{\infty}$ manifold, equipped with a properly Riemannian metric $e$, regular, that is (cf. [6]) we suppose $e$ is $C^{\infty}$, has an injectivity radius on $S$ (for the exponential map) strictly positive and has null curvature outside of a compact set. We call $H_{s}$ a space of tensor field $f$ over $S$, which are square integrable as well as their generalized covariant derivatives in the metric $e$ of order $k \leqq s$, which we denote by $\partial^{k} f$.

As in [3] we denote $\tilde{E}_{s}, E_{s}$ the (Banach) spaces of tensor fields over $S \times I$ :

$$
\tilde{E}_{s}(S \times I)=\bigcap_{1 \leqq k \leqq s} C^{k-1}\left(\bar{I}, H_{s-k}\right) \bigcap_{0 \leqq k \leqq s} W_{\infty}^{k}\left(I, H_{s-k}\right),
$$

and ( $G_{b}^{0}$ bounded continuous tensors)

$$
E_{s}(S \times I)=\left\{f \mid f \in C_{b}^{0}(S \times I), \quad \partial f \in \tilde{E}_{s-1}(S \times I)\right\} .
$$

Theorem 3. Let $g_{0}^{i j}, P_{0}^{i j}, \dot{P}_{0}^{i j}$ be Cauchy data on $S_{0}=S \times\{0\}$ such that $g_{0}^{i j} \in C_{b}^{0}$ is uniformly positive definite (with respect to $e$ ) on $S_{0}$ and:

$$
\partial g_{0}^{i j} \in H_{s-1}, \quad P_{0}^{i j} \in H_{s-1}, \quad \dot{P}_{0}^{i j} \in H_{s-2}, \quad \text { with } s \geqq 3 .
$$

Then: i) There exists an interval I such that the hyperbolic system (17), (18) admits a solution $g^{i j} \in E_{s}(S \times I)$ uniformly positive definite, $P^{i j} \in \tilde{E}_{s-1}$, having the prescribed initial data. This solution is unique.

(ii) If moreover the Cauchy data verify $\Sigma_{0}^{\alpha \beta}=0$ on $S_{0}$, then the solutions of (17), (18) also verifies $\Sigma^{\alpha \beta}=0$ on $S \times I$.

Proof. i) It is a theorem on hyperbolic equations, which may be obtained by refining the methods of Leray [13] in a manner analogous to the one used in [3]. The energy estimates for the linear case can be obtained in multiplying by a hyperbolic second order expression, with characteristic cone which separates the temporal axis from the isotropic cone.

ii) It is a consequence of the uniqueness of the solutions of the Cauchy problem for hyperbolic equations and of Theorem 2 ; the $\Sigma^{\alpha \beta}$ being zero for $x^{0}=0$ by hypothesis, and also $\dot{\Sigma}^{i 0}$ because of Bianchi's identity $\nabla_{0} \Sigma^{0 i}+\nabla_{j} \Sigma^{j i}=0$ considered at initial time.

Theorem 4. Let $(\boldsymbol{\gamma}, \mathbf{K})$ be initial data for Einstein equations on a regular riemannian manifold $(S, \mathbf{e})$. We suppose

$$
\begin{gathered}
\gamma \in C_{b}^{0} \text { and uniformly positive definite, } \\
\partial \gamma \in H_{s-1}, \quad \mathbf{K} \in H_{s-1}, \quad s \geqq 3 .
\end{gathered}
$$

Then, if the constraints are satisfied, there exists an Einstein space time $(S \times I, \mathbf{g})$, $\mathbf{g} \in E_{s}$, development of the initial data set $(S, \gamma, \mathbf{K})$.

Proof. It is an immediate consequence of Theorem 3, after remarking that, if we denote by $g_{0}^{i j}$ and $K_{0}^{i j}$ the image of the contravariant tensors associated with $\gamma$ and $\mathbf{K}$ by the diffeomorphism $S \rightarrow S_{0}=S \times\{0\} \subset S \times R$, and set

$$
\alpha_{0}=\left(e^{-1} g_{0}\right)^{1 / 2},
$$

the equation $\Sigma_{0}^{i j}=0$ (cf. [7]) determines $\dot{P}_{0}^{i j}$. 
Remark 1. The metric $\mathbf{g}$ constructed on $S \times I$ is of the form:

$$
d s^{2}=-e^{-1} g\left(d x^{0}\right)^{2}+g_{i j} d x^{i} d x^{j},
$$

with $g_{i j}$ uniformly bounded and positive definite with respect to $e_{i j}$. Thus $(S \times I, \mathbf{g})$ is globally hyperbolic, with $S_{0}$ a Cauchy surface (cf. [9]).

Remark 2. The geometrical (physical), global uniqueness theorem (cf. [9]) can also be proved in this context, but the proof seems to require more regularity than with the use of harmonic maps.

\section{Equivalent Hyperbolic Systems}

The lower terms $M^{i j}+H^{i j}$ that appear in (18) are extremely complicated, but if we choose $K_{i j}$ or $K_{j}^{i}$ as unknowns instead of $P^{i j}$, we obtain simpler hyperbolic systems. For instance let

$$
k_{i j}=\alpha K_{i j}, \quad a_{i}=\alpha^{-1} \partial_{i} \alpha, \quad f_{(i j)}=f_{i j}+f_{j i},
$$

if $\alpha=\left(e^{-1} g\right)^{1 / 2}$, we have :

$$
\begin{aligned}
\dot{g}_{i j}= & -2 k_{i j}, \\
& \square k_{i j}+3 k_{h(i} R_{j)}{ }^{h}-2 R_{i j}{ }^{h}{ }^{m} k_{h m}+2 k R_{i j}-2 a_{(i} D_{j)} k-4 k D_{i} a_{j} \\
& -k_{h(i} D_{j)} a^{h}+2 a^{h} D_{(i} k_{j) h}-a^{h} D_{h} k_{i j}-2 a^{h} D_{(i} k_{j) h}-3 k a_{i} a_{j} \\
& -4 \alpha^{-2} k_{i h} k_{j m} k^{h m} \\
\equiv & 2 k_{l(i}{ }^{(4)} R_{j)}^{l}+2 k^{(4)} R_{i j}-{ }^{(4)} \dot{R}_{i j}-D_{(i}\left\{\alpha^{2} \sum_{j j}^{0}\right\} .
\end{aligned}
$$

An expression using $K_{i}^{j}$ has been given in [10].

Acknowledgement. We thank C.N.R. (Gruppo Nazionale per la Fisica Matematica) and the University of Bologna whose hospitality has made our collaboration possible.

\section{References}

1. Arnowitt, R., Deser, S., Misner, C.: The dynamics of general relativity. In: Gravitation, an introduction to current research. Witten, L. (ed.). New York: Wiley 1962

2. Bergmann, P., Komar, A.: The phase space formulation of general relativity and approaches towards its canonical quantization. In: General relativity and gravitation. Held, A. (ed.). New York: Plenum Press 1980

3. Caricato, G.: Sul problema intriseco di evoluzione per le equazioni einsteiniani. Atti Ac. Naz. Lincei 41, 6, 487 (1966); 42, 1, 46 (1967)

4. Cattaneo, C.: Proiezioni naturali e derivazione trasversa in una varieta riemanniana a metrica iperbolica normale. Ann. Mat. Pura Appl. 48, 361 (1959)

5. Choquet-Bruhat, Y.: Théorème d'existence pour certains systèmes d'équations aux dérivées partielles non linéaires. Acta Mat. 88, 141 (1952)

6. Choquet-Bruhat, Y., Christodoulou, D., Francaviglia, M.: Cauchy data on a manifold. Ann. I.H.P. 29, (3), 241 (1978)

7. Choquet-Bruhat, Y., Segal, I.E.: Solution globale des équations de Yang-Mills sur l'univers d'Einstein. C.R. Acad. Sci. (Paris) 284, I (6), 225 (1982)

8. Choquet-Bruhat, Y.: Sur l'intégration des équations de la Relativité Générale. J. Rat. Mech. Anal. 5, 951 (1956)

9. Choquet,Bruhat, Y., York, J.: The Cauchy problem. In: General relativity and gravitation. Held, A. (ed.), Vol. I. New York: Plenum Press (survey article) 1980 
10. Choquet-Bruhat, Y., Ruggeri, T.: Hyperbolicité du système $3+1$ des équations d'Einstein. C.R. Acad. Sci. (Paris) 294, I, (12), 425 (1982)

11. Fischer, A.E., Marsden, J.E.: In: Isolated gravitating systems in general relativity. Ehlers, J. (ed.), p. 322. Amsterdam: North-Holland 1976

12. Hughes, J., Kato, J., Marsden, J.: Well-posed quasi-linear second order hyperbolic systems with applications to non-linear elastodynamics and general relativity. Arch. Rat. Mech. Anal. 63, 273 (1976)

13. Leray, J.: Hyperbolic differential equations. Institute of Advanced Studies (1952)

14. Lichnerowicz, A.: Propagateurs et commutateurs en relativité générale. I.H.E.S. Vol. 10 (1961)

15. Lichnerowicz, A.: Problèmes globaux en Mécanique Relativiste. Paris: Hermann 1939

16. Smarr, L., York, J.: Radiation gauge in general relativity. Phys. Rev. D 17, (2), 1945 (1978)

17. Segal, I.E.: The Cauchy problem for the Yang-Mills equations. J. Funct. Anal. 33, 175-194 (1979)

18. Eardley, D., Moncrief, V.: The global existence of Yang-Mills fields in 4-dimensional space-time, Parts I and II. Commun. Math. Phys. 83, 171-212 (1982)

Communicated by S.-T. Yau

Received October 21, 1982 
